\title{
PORTFOLIO OPTIMIZATION WITH DRAWDOWN CONSTRAINTS
}

\author{
Alexei Chekhlov ${ }^{1}$, Stanislav Uryasev ${ }^{2}$, Michael Zabarankin ${ }^{2}$ \\ Risk Management and Financial Engineering Lab \\ Center for Applied Optimization \\ Department of Industrial and Systems Engineering \\ University of Florida, Gainesville, FL 32611
}

Date: January 13, 2003

Correspondence should be addressed to: Stanislav Uryasev

\begin{abstract}
We propose a new one-parameter family of risk functions defined on portfolio return sample-paths, which is called conditional drawdown-at-risk (CDaR). These risk functions depend on the portfolio drawdown (underwater) curve considered in active portfolio management. For some value of the tolerance parameter $\alpha$, the $\mathrm{CDaR}$ is defined as the mean of the worst $(1-\alpha) * 100 \%$ drawdowns. The CDaR risk function contains the maximal drawdown and average drawdown as its limiting cases. For a particular example, we find optimal portfolios with constraints on the maximal drawdown, average drawdown and several intermediate cases between these two. The $\mathrm{CDaR}$ family of risk functions originates from the conditional value-at-risk $(\mathrm{CVaR})$ measure. Some recommendations on how to select the optimal risk measure for getting practically stable portfolios are provided. We solved a real life portfolio allocation problem using the proposed risk functions.
\end{abstract}

\section{Introduction}

Optimal portfolio allocation is a longstanding issue in both practical portfolio management and academic research on portfolio theory. Various methods have been proposed and studied (for a review, see, for example, Grinold and Kahn, 1999). All of them, as a starting point, assume some measure of portfolio risk.

From a standpoint of a fund manager, who trades clients' or bank's proprietary capital, and for whom the clients' accounts are the only source of income coming in the form of management and incentive fees, losing these accounts is equivalent to the death of his business. This is true with no regard to whether the employed strategy is long-term valid and has very attractive expected

\footnotetext{
${ }^{1}$ Thor Asset Management, Inc., 551 Fifth Ave., Suite 601, 6th Floor, New York, NY 10017; e-mail: a chekhlov@thorcapital.com

${ }^{2}$ University of Florida, ISE, P.O. Box 116595, 303 Weil Hall Gainesville, FL 32611-6595; e-mail: uryasev@ise.ufl.edu, zabarank@ufl.edu
} 
return characteristics. Such fund manager's primary concern is to keep the existing accounts and to attract the new ones in order to increase his revenues. A particular client who was persuaded into opening an account with the manager through reading the disclosure document, listening to the manager's attractive story, knowing his previous returns, etc., will decide on firing the manager based, most likely, on his account's drawdown magnitude and duration. In particular, it is highly uncommon, for a Commodity Trading Advisor (CTA) to still hold a client whose account was in a drawdown, even of small size, for longer than 2 years. By the same token, it is unlikely that a particular client will tolerate a 50\% drawdown in an account with an average- or small-risk CTA. Similarly, in an investment bank setup, a proprietary system trader will be expected to make money in 1 year at the longest, i.e. he cannot be in a drawdown for longer than a year. Also, he/she may be shut down if a certain maximal drawdown condition will be breached, which, normally, is around $20 \%$ of his backing equity. Additionally, he will be given a warning drawdown level at which he will be reviewed for letting him keep running the system (around 15\%). Obviously, these issues make managed accounts practitioners very concerned about both the magnitude and duration of their clients' accounts drawdowns.

First, we want to mention the paper by Grossman and Zhou, 1993, where an assumption of lognormality of equity statistics and use of dynamic programming theory led to an exact analytical solution of a maximal drawdown problem for a one-dimensional case. A subsequent generalization of this work for multiple dimensions was done in Cvitanic and Karatzas, 1995. In difference to these works, which find time-dependent portfolio allocation strategies under some assumptions on distributions of portfolio returns, this paper defines risk and finds optimal weights (constant over time) for a single sample-path of portfolio returns. We will be looking to find a constant set of weights, which will satisfy a certain risk condition over a period of time. We make no assumption about the underlying probability distribution, which allows considering variety of practical applications. We concentrate on the portfolio equity curves over a particular sample-path (historical or most probable future sample-path), i.e. we define some sample-path risk function rather than a risk measure on set of sample-paths. Being perfectly aware of this insufficiency, we leave the issue of definition and optimization of the drawdown-type risk measures on a set of sample-paths for future research. Also, we do not investigate the predictive power of a constant set of weights, trying to introduce and test the new approach in a simplified version. To some extend, we consider a setup similar to the index tracking problem, considered by Dembo and King, 1992, where an index historical performance is replicated by a portfolio with constant weights.

In this paper, we have introduced and studied a one-parameter family of risk functions called conditional drawdown-at-disk (CDaR). This risk function quantifies in aggregated format the frequency and magnitude of the portfolio drawdowns over some period of time for a particular sample-path. By definition, a drawdown is the drop in the portfolio value comparing to the maximum achieved in the past. We can define drawdown in absolute or relative (percentage) terms. For example, if at the present time the portfolio value equals $\$ 9 \mathrm{M}$ and the maximal portfolio value in the past was $\$ 10 \mathrm{M}$, we can say that the portfolio drawdown in absolute terms equals $\$ 1 \mathrm{M}$ and in relative terms equals $10 \%$. For some value of the confidence parameter $\alpha$, the $\alpha-\mathrm{CDaR}$ is defined as the mean of the worst $(1-\alpha) * 100 \%$ drawdowns experienced over some period of time on a sample-path. For instance, $0.95-\mathrm{CDaR}$ (or 95\%- CDaR) is the average of the worst $5 \%$ drawdowns over the considered time interval. The CDaR risk function contains the average drawdown and maximal drawdown as its limiting cases. It takes into account both the magnitude and duration of the drawdowns, whereas the maximal drawdown function concentrates on a single event - maximal portfolio loss compared to its previous highest value. 
The CDaR risk function is related to Value-at-Risk (VaR) risk measure and to Conditional Valueat-Risk (CVaR) risk measure studied in the paper by Rockafellar and Uryasev, 2000; 2002. By definition, with a specified probability level $\alpha$, the $\alpha-V a R$ of a portfolio is the lowest amount $\zeta_{\alpha}$ such that the probability that the loss will not exceed $\zeta_{\alpha}$ is greater or equal to $\alpha$, in a specified time $\tau$ (Jorion, 1996), whereas the $\alpha-\mathrm{CVaR}$ is $\alpha$-tail expectation of losses (Rockafellar and Uryasev, 2000; 2002). For continuous distributions, $\alpha$-tail expectation of losses coincides with the conditional expectation of losses above the VaR value $\zeta_{\alpha}$.

The CDaR risk function is based on CVaR concept and can be viewed as a modification of the $\mathrm{CVaR}$ to the case when the loss-function is defined as a drawdown for discrete time moments having equal weights (instead of scenario probabilities). $\mathrm{CDaR}$ and $\mathrm{CVaR}$ are conceptually closely related percentile-based functions. The optimization approach developed for CVaR by Rockafellar and Uryasev, 2000; 2002 can be directly extended to CDaR optimization. A nice feature of the CVaR optimization approach is that the resulting problem is linear in an extended set of variables, and the VaR value, $\zeta_{\alpha}$, is calculated automatically using an auxiliary variable (Rockafellar and Uryasev, 2000; 2002). The paper by Krokhmal et al., 2002, considers several equivalent approaches for generating return- $\mathrm{CVaR}$ efficient frontiers; in particular, it considers an approach, which maximizes return with $\mathrm{CVaR}$ constraints.

In the case of continuous distributions, CVaR is known also as Mean Excess Loss, Mean Shortfall (Mausser and Rosen, 1999), or Tail Value-at-Risk (Artzner et al., 1999). A case study on the hedging of a portfolio of options using the CVaR minimization technique is included in Rockafellar and Uryasev, 2000. Also, the CVaR minimization approach was applied to credit risk management of a portfolio of bonds (Andersson et al., 1999). A case study on optimization of a portfolio of stocks with CVaR constraints is considered in Krokhmal et al., 2002.

Similar to the Markowitz mean-variance approach (Markowitz, 1952) in a sample-variance setting, we formulate and solve the optimization problem with the return performance function and $\mathrm{CDaR}$ constraints. The return-CDaR optimization problem is a convex optimization problem with a linear performance function and piece-wise linear convex constraint (see definition of convexity in Rockafellar, 1970). This paper reduces the return-CDaR optimization problem to a linear programming problem. Linear programming allows solving optimization problems with many thousands of instruments. The algorithm is fast, numerically stable, and provides a solution during one run (without adjusting parameters like in genetic algorithms or neural networks). Linear programming approaches are routinely used in portfolio optimization with various criteria, including mean absolute deviation (Konno and Yamazaki, 1991), maximum deviation (Young, 1998), and mean regret (Dembo and King, 1992). A reader interested in other applications of optimization techniques in the finance area can find relevant papers in Ziemba and Mulvey, 1998.

\section{General Setup}

Denote by function $w(x, t)$ the uncompounded portfolio return at time $t$, where the components of the portfolio vector $x=\left(x_{1}, x_{2}, \ldots, x_{m}\right)$ are weights of $m$ instruments in the portfolio. The drawdown function at time $t$ is defined as the difference between the maximum of the function $w(x, t)$ over the history preceding time $t$ and the value of this function at time $t$

$$
D(x, t)=\max _{0 \leq \tau \leq t}\{w(x, \tau)\}-w(x, t) .
$$


We consider three risk functions defined on a sample-path of portfolio returns: (i) maximum drawdown (MaxDD), (ii) average drawdown (AvDD), and (iii) conditional drawdown-at-risk $(\mathrm{CDaR})$. The $\mathrm{CDaR}$ risk function is actually a family of risk functions parameterized by the parameter $\alpha$. It contains, as a special case, the maximum drawdown and average drawdown risk functions.

The maximum drawdown on the interval $[0, T]$, is calculated by maximizing the drawdown function $D(x, t)$, i.e.

$$
\mathbf{M}(x)=\max _{0 \leq t \leq T}\{D(x, t)\}
$$

The average drawdown is equal to

$$
\mathbf{A}(x)=\frac{1}{T} \int_{0}^{T} D(x, t) d t
$$

Let $N$ denote the number of time sub-periods in the time interval $[0, T]$ and $\alpha \in[0,1]$ denote the confidence level. In a case when $(1-\alpha) N$ is an integer number (i.e. we are able to precisely count $(1-\alpha) * 100 \%$ of the drawdowns), the $\mathrm{CDaR}$ is defined as the mean of the worst $(1-\alpha) * 100 \%$ drawdowns. For instance, if $\alpha=0, \mathrm{CDaR}$ equals the average drawdown over all sub-periods, and if $\alpha=0.95$, then CDaR is the average of the worst $5 \%$ drawdowns. Let $\zeta_{\alpha}(x)$ denote a threshold such that exactly $(1-\alpha) * 100 \%$ of drawdowns exceed this threshold (it is supposed that $(1-\alpha) N$ is an integer number). In this case, $\mathrm{CDaR}$ with a confidence level $\alpha$ is the average of $(1-\alpha) * 100 \%$ of the drawdowns

$$
\Delta_{\alpha}(x)=\frac{1}{(1-\alpha) T} \int_{\Omega_{\alpha}} D(x, t) d t,
$$

where $\Omega_{\alpha}=\left\{t \in[0, T]: D(x, t) \geq \zeta_{\alpha}(x)\right\}$. However, when $(1-\alpha) N$ is not integer, which $\dot{\mathrm{s}}$ a general case, $\mathrm{CDaR}$ is expressed as a liner combination of $\zeta_{\alpha}(x)$ and drawdowns strictly exceeding $\zeta_{\alpha}(x)$, similar as it was done for CVaR by Rockafellar and Uryasev, 2002. The following optimization formula presents $\mathrm{CDaR}$ in a general case

$$
\Delta_{\alpha}(x)=\min _{\zeta}\left\{\zeta+\frac{1}{(1-\alpha) T} \int_{0}^{T}[D(x, t)-\zeta]^{+} d t\right\},
$$

where $[g]^{+}=\max \{0, g\}$. If $(1-\alpha) N$ is integer, then optimal value of $\zeta$ in (4) coincides with $\zeta_{\alpha}(x)$, otherwise optimal $\zeta$ is greater than or equal to $\zeta_{\alpha}(x)$.

When $\alpha$ tends to $1, \mathrm{CDaR}$ tends to the maximum drawdown, i.e. $\Delta_{1}(x)=\mathbf{M}(x)$, and when $\alpha=0, \mathrm{CDaR}$ coincides with the average drawdown, i.e. $\Delta_{0}(x)=\mathbf{A}(x)$.

In portfolio optimization problems, depending on our risk preference, we can impose constraints on the maximum drawdown given by (2)

$$
\mathbf{M}(x) \leq \mathrm{v}_{1} C,
$$

on average drawdown given by (3) 


$$
\mathbf{A}(x) \leq \mathrm{v}_{2} C,
$$

on $\mathrm{CDaR}$ given by (4)

$$
\Delta_{\alpha}(x) \leq v_{3} C,
$$

or combine several constraints together

$$
\mathbf{M}(x) \leq \mathrm{v}_{1} C, \quad \mathbf{A}(x) \leq \mathrm{v}_{2} C, \quad \Delta_{\alpha}(x) \leq \mathrm{v}_{3} C,
$$

where the constant $C$ represents the available capital and coefficients $v_{1}, v_{2}$ and $v_{3}$ define the capital portions "allowed to be lost". Usually,

$$
0 \leq \mathrm{v}_{1}, \mathrm{v}_{2}, \mathrm{v}_{3} \leq 1 .
$$

Let $j$ be an instrument index, $1 \leq j \leq m$. Suppose that for a particular sample-path, returns $\left(r_{j}(1), r_{j}(2), \ldots, r_{j}(N)\right), 1 \leq j \leq m$, for the portfolio instruments are available. Denote by $y_{j}(t)=\sum_{k=1}^{t} r_{j}(k)$ the uncompounded cumulative return of the $j^{\text {th }}$ instrument up to the time moment $t$. Let the vector $y(t)=\left(y_{1}(t), y_{2}(t), \ldots, y_{m}(t)\right)$ be a set of uncompounded cumulative returns for $m$ portfolio instruments at time moment $t$. The cumulative portfolio return equals $w(x, t)=y(t) \cdot x=\sum_{i=1}^{m} y_{i}(t) x_{i}$.

The average annualized return $R(x)$ over the period $[0, T]$, which is a linear function of $x$, is defined as follows

$$
R(x)=\frac{1}{d C} w(x, N)=\frac{1}{d C} y(N) \cdot x,
$$

where $d$ is the number of years in the time interval $[0, T]$ and $C$ is the available capital.

We impose the so-called technological constraints on the vector $x$ given by box inequalities

$$
X=\left\{x: x_{\min } \leq x_{i} \leq x_{\max }, \quad i=\overline{1, m}\right\}
$$

for some constant values of $x_{\min }$ and $x_{\max }$. However, other feasible constraints can be imposed. In the next section, we will consider the problem of maximizing return $R(x)$ subject to constraints on various risk functions and technological constraints (8) on the portfolio positions.

\section{Portfolio Optimization with Drawdown Constraints}

The maximization of the average return with constraints on maximum drawdown (see, (9)), average drawdown (see, (10)), or $\mathrm{CDaR}$ (see, (11)) can be formulated as the following mathematical programming problems:

$$
\begin{array}{ll}
\max _{x \in X} & R(x) \\
\text { s.t. } & \mathbf{M}(x) \leq \mathrm{v}_{1} C,
\end{array}
$$




$$
\begin{array}{ll}
\max _{x \in X} & R(x) \\
\text { s.t. } & \mathbf{A}(x) \leq \mathrm{v}_{2} C, \\
\max _{x \in X} & R(x) \\
\text { s.t. } & \Delta_{\alpha}(x) \leq \mathrm{v}_{3} C .
\end{array}
$$

Similar to Rockafellar and Uryasev, 2000; 2002, problems (9), (10) and (11) can be reduced to linear programming problems using auxiliary variables.

\section{Discrete Formulation: Reduction to Linear Programming Problems}

Denote the vector of cumulative instrument returns up to the time moment $k$ by

$$
y_{k}=y(k) \text {, }
$$

then the drawdown function is defined by the expression

$$
D_{k}(x)=\max _{1 \leq j \leq k}\left\{y_{j} \cdot x\right\}-y_{k} \cdot x,
$$

and the average annualized return function is given by the inner product

$$
R(x)=\frac{1}{d C} y_{N} \cdot x .
$$

Problems (9), (10) and (11) are formulated accordingly.

The optimization problem with a constraint on maximum drawdown is

$$
\begin{array}{ll}
\max _{x \in X} & \frac{1}{d C} y_{N} \cdot x \\
\text { s.t. } & \max _{1 \leq k \leq N}\left\{\max _{1 \leq j \leq k}\left\{y_{j} \cdot x\right\}-y_{k} \cdot x\right\} \leq \mathrm{v}_{1} C,
\end{array}
$$

which is reduced to the following linear programming (LP) problem

$$
\begin{aligned}
\max _{x, u} & \frac{1}{d C} y_{N} \cdot x \\
\text { s.t. } & u_{k}-y_{k} \cdot x \leq v_{1} C, \quad 1 \leq k \leq N, \\
& u_{k} \geq y_{k} \cdot x, \quad 1 \leq k \leq N, \\
& u_{k} \geq u_{k-1}, \quad 1 \leq k \leq N, \\
& u_{0}=0, \\
& x_{\min } \leq x_{i} \leq x_{\max }, \quad 1 \leq i \leq m,
\end{aligned}
$$

where $u_{k}, 1 \leq k \leq N$, are auxiliary variables.

The optimization problem with a constraint on average drawdown can be written as follows 


$$
\begin{array}{ll}
\max _{x \in X} & \frac{1}{d C} y_{N} \cdot x \\
\text { s.t. } & \frac{1}{N} \sum_{k=1}^{N}\left(\max _{1 \leq j \leq k}\left\{y_{j} \cdot x\right\}-y_{k} \cdot x\right) \leq v_{2} C,
\end{array}
$$

or, equivalently,

$$
\begin{array}{ll}
\max _{x, u} & \frac{1}{d C} y_{N} \cdot x \\
\text { s.t. } & \frac{1}{N} \sum_{k=1}^{N}\left(u_{k}-y_{k} \cdot x\right) \leq v_{2} C, \\
& u_{k} \geq y_{k} \cdot x, \quad 1 \leq k \leq N, \\
& u_{k} \geq u_{k-1}, \quad 1 \leq k \leq N, \\
& u_{0}=0, \\
& x_{\min } \leq x_{i} \leq x_{\max }, \quad 1 \leq i \leq m .
\end{array}
$$

Following the approach for Conditional Value-at-Risk (CVaR), see Rockafellar and Uryasev, 2002 , it can be proved that the optimization problem with a constraint on CDaR may be formulated as follows

$$
\begin{aligned}
& \max _{x \in X} \frac{1}{d C} y_{N} \cdot x \\
& \text { s.t. } \quad \zeta+\frac{1}{(1-\alpha) N} \sum_{k=1}^{N}\left[\max _{1 \leq j \leq k}\left\{y_{j} \cdot x\right\}-y_{k} \cdot x-\zeta\right]^{+} \leq v_{3} C,
\end{aligned}
$$

which is reduced to the LP

$$
\begin{array}{ll}
\max _{x, \zeta, u, z} & \frac{1}{d C} y_{N} \cdot x \\
\text { s.t. } & \zeta+\frac{1}{(1-\alpha) N} \sum_{k=1}^{N} z_{k} \leq v_{3} C, \\
& z_{k} \geq u_{k}-y_{k} \cdot x-\zeta, \quad 1 \leq k \leq N, \\
& z_{k} \geq 0, \quad 1 \leq k \leq N, \\
& u_{k} \geq y_{k} \cdot x, \quad 1 \leq k \leq N, \\
& u_{k} \geq u_{k-1}, \quad 1 \leq k \leq N, \\
& u_{0}=0, \\
& x_{\min } \leq x_{i} \leq x_{\max }, \quad 1 \leq i \leq m .
\end{array}
$$

An important feature of formulation (18) is that it does not involve the threshold function $\zeta_{\alpha}(x)$. At optimal solution of problem (18), variables $x$ and $\zeta$ give an optimal portfolio and corresponding value of the threshold function. 


\section{Calculation Results}

For numerical experiments, we have considered the equity curves generated by a futures technical trading system in $m=32$ different markets (a wide range of major liquid markets: currencies, currency crosses, U.S. treasuries both short- and long-term, foreign long-term treasuries, international equity indices, and metals). The list of market ticker symbols, provided in the results below, is mnemonic and corresponds to the widely used data provider, FutureSource. The individual equity curves covered a time span of 1/1/1988 through 9/1/1999. The equity curves were based on $\$ 20 \mathrm{M}$ backing equity in a margin account and were uncompounded, i.e. the risk was taken based always on the original \$20M and did not account for the money made or lost during a trading strategy.

As for the technological constraints (8), we chose $x_{\min }=0.2$ and $x_{\max }=0.8$. This choice was dictated by the need to have the resultant margin-to-equity ratio in the account within admissible bounds, which are specific for a particular portfolio. In this futures trading setup, these constraints are analogous to the "fully-invested" condition from classical Sharpe-Markowitz theory. They define bounds on the leverage of the strategy and make an efficient frontier to be concave. If all positions are equal to the lower bound 0.2 , then the sum of the positions equals $0.2 \cdot 32=6.4$ and the minimal leverage equals 6.4 . However, if all positions are equal to the upper bound 0.8 , then the sum of the positions equals $0.8 \cdot 32=25.6$ and the maximal leverage equals 25.6. The optimal allocation of weights picks both the optimal leverage and proportions between instruments.

The absence of technological constraints (8) leads to the infinite leverage of these types of strategies and as a result, an efficient frontier would be a straight line passing through $(0,0)$. Another subtle issue is the stability of the optimal portfolios in the case when the constraints are "too lax". It is a matter of empirical evidence that the more lax the constraints are - the better portfolio equity curve you can get through optimal mixing - and the less stable these results would be with respect to walk-forward analysis. The aforementioned set of constraints was empirically found to be both leading to sufficiently stable portfolios and allowing enough mix of the individual equity curves.

The problem, then, is to find a set of weights $x=\left(x_{1}, x_{2}, \ldots, x_{m}\right)$, such that it solves the minimization problems (16), (17), or (18). These linear programming problems have been solved by the CPLEX solver (inputs are prepared with $\mathrm{C}++$ programming language). An alternative verification of the solutions was obtained via solving these optimization problems by a genetic algorithm implemented in VB6, discussion of which is beyond the present scope.

Let us denote (16), (17) and (18) by MaxDD, AvDD and $\alpha-\mathrm{CDaR}$ problems, respectively. We have solved these optimization problems for cases of $(1-\alpha)=0,0.05,0.1,0.2,0.4,0.6,0.8$ and 1. As we have noted before, cases of $(1-\alpha)=0$ and $(1-\alpha)=1$ correspond to MaxDD and AvDD problems, accordingly.

Tables 1 and 2 present the list of markets and corresponding sets of optimal weights for MaxDD and AvDD problems. Table 3 presents the weights for the CDaR case with the confidence level $(1-\alpha)=0.05$. In these tables, the solution achieving maximal reward/risk ratio is boldfaced.

Note that the smallest value of risk is chosen in such a way that the solutions of the optimization problem still exist. This means that each problem does not have a solution beyond the upper and 
lower bounds of the risk range covered (the whole efficient frontier is shown). The risk and rate of return are expressed in percentage terms with respect to the original account size, i.e. \$20M.

The efficient frontiers for the reward-MaxDD and reward-AvDD problems are shown in Figures 1 and 2, respectively. We do not show efficient frontiers for reward-CDaR problem in separate graphs (except for MaxDD and AvDD). However, in Figure 3 we show the reward-MaxDD graphs for portfolios optimal with $(1-\alpha)=0,0.05,0.4$ and $1 \mathrm{CDaR}$ constraints. As expected, the case with $(1-\alpha)=0 \mathrm{CDaR}$ corresponding to MaxDD has a concave efficient frontier dominating other graphs. The reward is not maximal for each level of MaxDD when we solved the optimization problems with $(1-\alpha)=0.05,0.4$ and $1 \mathrm{CDaR}$ constraints. Viewed from the reference point of MaxDD problem, $(1-\alpha)<1$ solutions are uniformly "worse." However, none of these solutions are truly better or worse than others from a mathematical standpoint. Each of them provides the optimal solution in its own sense. Some thoughts on quality of solutions are discussed from a practical standpoint below. Similar to Figure 3, Figure 4 depicts the rewardAvDD graphs for portfolios optimal with $(1-\alpha)=0,0.05,0.4$ and $1 \mathrm{CDaR}$ constraints. The case with $(1-\alpha)=1 \mathrm{CDaR}$ corresponding to AvDD has a concave efficient frontier dominating other graphs.

As in classical portfolio theory, we are interested in a portfolio with a maximal reward/risk ratio, i.e. the portfolio corresponding to the point of contact of the straight line coming through $(0,0)$ and the efficient frontier. For the risk defined in terms of problems (16), (17), and (18), we will call the reward/risk ratios as MaxDDRatio, AvDDRatio, and CDaRRatio which, by definition, are:

$$
\text { MaxDDRatio }=R(x) / \mathbf{M}(x), \text { AvDDRatio }=R(x) / \mathbf{A}(x) \text {, and CDaRRatio }=R(x) / \Delta_{\alpha}(x) .
$$

The charts of MaxDDRatio and AvDDRatio quantities are shown in Figures 5 and 6 for the same cases of $(1-\alpha)$ as in Figures 3 and 4.

We have solved optimization problem (18) for cases of $(1-\alpha)=0,0.05,0.1,0.2,0.4,0.6,0.8$ and 1 . Let us note that the case of $(1-\alpha)=0.05$ (see Table 3 ), considering minimization of the worst $5 \%$ part of the underwater curve, produces a set of weights significantly different from the $(1-\alpha)=0$ case (MaxDD problem), and $(1-\alpha)=0.05 \mathrm{CDaR}$ case contains several tens of events over which the averaging was performed. We consider that optimization with $(1-\alpha)=0.05$ or 0.1 constraints produces a more robust portfolio than the optimization with MaxDD or AvDD constraints. CDaR solution takes into account many significant drawdowns, comparing to MaxDD considering only the largest drawdown. Also, CDaR solution is not impacted by many small drawdowns like a solution in AvDD case.

We have also verified our results via solving the related nonlinear optimization problems corresponding to problems (16-18). These problems have optimized the drawdown ratios corresponding to the same set of constraints. Verification was done using a genetic optimization algorithm. We were satisfied to find that this procedure has produced the same sets of weights for the optimal solutions. 


\section{Conclusions}

We have introduced a new $\mathrm{CDaR}$ risk function, which is quite useful in practical portfolio management. This function is based on $\mathrm{CVaR}$ risk measure and has the MaxDD and AvDD risk functions as its limiting cases. We have studied reward/risk ratios implied by these risk functions, namely MaxDDRatio, AvDDRatio, and CDaRRatio. We have shown that the portfolio allocation problem with CDaR, MaxDD and AvDD risk functions can be efficiently solved. We have formulated and solved a portfolio allocation problem for a real-life example. Implemented in a managed accounts' environment, these developments will allow a trading or risk manager to allocate risk according to his/her personal assessment of magnitude and duration of extreme drawdowns of a portfolio equity.

We think that solutions produced by the MaxDD approach may contain a significant amount of statistical error since it is based on a single observation of maximal loss. The CDaR family of risk functions allows a risk manager to control the worst $(1-\alpha) * 100 \%$ drawdowns. Due to the statistical averaging of drawdowns, a better prediction of risk in the future and a more stable portfolio can be obtained. Our studies indicate that CDaR control with an appropriate level (e.g. $\alpha=0.95$, i.e. optimizing over the $5 \%$ of the worst drawdowns) produces a more stable weight allocation compared to one produced by MaxDD control. A detailed study of this issue calls for a separate publication. 
Table 1. List of markets and corresponding sets of optimal weights for the MaxDD problem. The solution achieving maximal reward/risk ratio is boldfaced.

\begin{tabular}{|l|c|c|c|c|c|c|c|c|c|c|c|c|c|c||}
\hline Risk, \% & 4.0 & 5.0 & $\mathbf{6 . 0}$ & 7.0 & 8.0 & 9.0 & 10.0 & 11.0 & 12.0 & 13.0 & 14.0 & 15.0 & 16.0 & 17.0 \\
\hline Reward, \% & 25.0 & 36.3 & $\mathbf{4 4 . 5}$ & 51.4 & 57.3 & 63.0 & 67.7 & 71.7 & 75.2 & 78.0 & 80.4 & 81.9 & 82.9 & 83.0 \\
\hline Reward/Risk & 6.26 & 7.27 & $\mathbf{7 . 4 2}$ & 7.34 & 7.16 & 7.00 & 6.77 & 6.52 & 6.27 & 6.00 & 5.74 & 5.46 & 5.18 & 4.88 \\
\hline \hline
\end{tabular}

\section{OPTIMAL PORTFOLIO CONFIGURATION}

\begin{tabular}{|c|c|c|c|c|c|c|c|c|c|c|c|c|c|c|}
\hline AAO & 0.20 & 0.25 & 0.25 & 0.28 & 0.21 & 0.39 & 0.68 & 0.80 & 0.69 & 0.80 & 0.80 & 0.80 & 0.80 & 0.80 \\
\hline AD & 0.20 & 0.40 & 0.74 & 0.80 & 0.80 & 0.80 & 0.80 & 0.80 & 0.80 & 0.80 & 0.80 & 0.80 & 0.80 & 0.80 \\
\hline$\overline{\text { AXB }}$ & 0.20 & 0.37 & 0.32 & 0.47 & 0.63 & 0.80 & 0.55 & 0.64 & 0.80 & 0.80 & 0.80 & 0.80 & 0.80 & 0.80 \\
\hline$\overline{B D}$ & 0.20 & 0.20 & 0.20 & 0.20 & 0.62 & 0.41 & 0.53 & 0.56 & 0.80 & 0.80 & 0.80 & 0.80 & 0.80 & 0.80 \\
\hline$\overline{\mathbf{B P}}$ & 0.20 & 0.20 & 0.20 & 0.20 & 0.20 & 0.20 & 0.20 & 0.20 & 0.22 & 0.51 & 0.77 & 0.80 & 0.80 & 0.80 \\
\hline$\overline{C D}$ & 0.25 & 0.59 & 0.80 & 0.80 & 0.80 & 0.80 & 0.80 & 0.80 & 0.80 & 0.80 & 0.80 & 0.80 & 0.80 & 0.80 \\
\hline $\mathbf{C P}$ & 0.62 & 0.80 & 0.77 & 0.80 & 0.80 & 0.80 & 0.80 & 0.80 & 0.80 & 0.80 & 0.80 & 0.80 & 0.80 & 0.80 \\
\hline DGB & 0.20 & 0.80 & 0.80 & 0.80 & 0.80 & 0.80 & 0.80 & 0.80 & 0.80 & 0.80 & 0.80 & 0.80 & 0.80 & 0.80 \\
\hline DX & 0.20 & 0.20 & 0.20 & 0.20 & 0.20 & 0.20 & 0.20 & 0.20 & 0.63 & 0.80 & 0.80 & 0.80 & 0.80 & 0.80 \\
\hline ED & 0.20 & 0.20 & 0.20 & 0.20 & 0.20 & 0.20 & 0.20 & 0.20 & 0.20 & 0.35 & 0.74 & 0.80 & 0.80 & 0.80 \\
\hline$\overline{E U}$ & 0.20 & 0.20 & 0.20 & 0.80 & 0.80 & 0.80 & 0.80 & 0.80 & 0.80 & 0.80 & 0.80 & 0.80 & 0.80 & 0.80 \\
\hline FV & 0.20 & 0.20 & 0.39 & 0.58 & 0.52 & 0.50 & 0.54 & 0.80 & 0.80 & 0.80 & 0.80 & 0.80 & 0.80 & 0.80 \\
\hline FXADJY & 0.27 & 0.58 & 0.77 & 0.80 & 0.80 & 0.80 & 0.80 & 0.80 & 0.80 & 0.80 & 0.80 & 0.80 & 0.80 & 0.80 \\
\hline FXBPJY & 0.20 & 0.20 & 0.20 & 0.20 & 0.20 & 0.20 & 0.53 & 0.80 & 0.80 & 0.80 & 0.80 & 0.80 & 0.80 & 0.80 \\
\hline FXEUBP & 0.20 & 0.28 & 0.29 & 0.32 & 0.34 & 0.65 & 0.72 & 0.80 & 0.80 & 0.80 & 0.80 & 0.80 & 0.80 & 0.80 \\
\hline FXEUJY & 0.20 & 0.20 & 0.41 & 0.80 & 0.80 & 0.80 & 0.80 & 0.80 & 0.80 & 0.80 & 0.80 & 0.80 & 0.80 & 0.80 \\
\hline FXEUSF & 0.33 & 0.20 & 0.25 & 0.30 & 0.73 & 0.80 & 0.80 & 0.80 & 0.80 & 0.80 & 0.80 & 0.80 & 0.80 & 0.80 \\
\hline FXNZUS & 0.20 & 0.20 & 0.20 & 0.20 & 0.20 & 0.20 & 0.20 & 0.20 & 0.20 & 0.20 & 0.20 & 0.27 & 0.80 & 0.80 \\
\hline FXUSSG & 0.20 & 0.20 & 0.20 & 0.20 & 0.20 & 0.20 & 0.20 & 0.28 & 0.21 & 0.43 & 0.72 & 0.80 & 0.80 & 0.80 \\
\hline FXUSSK & 0.20 & 0.80 & 0.80 & 0.65 & 0.73 & 0.70 & 0.60 & 0.35 & 0.20 & 0.20 & 0.20 & 0.80 & 0.80 & 0.80 \\
\hline GC & 0.20 & 0.20 & 0.20 & 0.20 & 0.20 & 0.20 & 0.20 & 0.20 & 0.20 & 0.20 & 0.20 & 0.57 & 0.80 & 0.80 \\
\hline JY & 0.20 & 0.23 & 0.34 & 0.25 & 0.37 & 0.80 & 0.80 & 0.80 & 0.80 & 0.80 & 0.80 & 0.80 & 0.80 & 0.80 \\
\hline LBT & 0.20 & 0.35 & 0.62 & 0.80 & 0.80 & 0.80 & 0.80 & 0.80 & 0.80 & 0.80 & 0.80 & 0.80 & 0.80 & 0.80 \\
\hline LFT & 0.20 & 0.20 & 0.20 & 0.20 & 0.39 & 0.63 & 0.80 & 0.80 & 0.80 & 0.80 & 0.80 & 0.80 & 0.80 & 0.80 \\
\hline LGL & 0.20 & 0.20 & 0.20 & 0.20 & 0.20 & 0.20 & 0.20 & 0.37 & 0.80 & 0.80 & 0.80 & 0.80 & 0.80 & 0.80 \\
\hline LML & 0.20 & 0.27 & 0.36 & 0.46 & 0.51 & 0.60 & 0.78 & 0.80 & 0.80 & 0.80 & 0.80 & 0.80 & 0.80 & 0.80 \\
\hline$\overline{\text { MNN }}$ & 0.20 & 0.30 & 0.42 & 0.45 & 0.44 & 0.80 & 0.80 & 0.80 & 0.77 & 0.80 & 0.80 & 0.80 & 0.80 & 0.80 \\
\hline SF & 0.20 & 0.20 & $\begin{array}{l}0.37 \\
\end{array}$ & 0.39 & 0.52 & 0.52 & 0.63 & 0.75 & 0.80 & 0.80 & 0.80 & 0.80 & 0.80 & 0.80 \\
\hline SI & 0.20 & 0.20 & 0.20 & 0.20 & 0.20 & 0.20 & 0.20 & 0.20 & 0.20 & 0.20 & 0.20 & 0.20 & 0.40 & 0.80 \\
\hline SJB & 0.49 & 0.74 & 0.80 & 0.80 & 0.80 & 0.80 & 0.80 & 0.80 & 0.80 & 0.80 & 0.80 & 0.80 & 0.80 & 0.80 \\
\hline SNI & 0.20 & 0.56 & 0.67 & 0.69 & 0.78 & 0.80 & 0.80 & 0.80 & 0.80 & 0.80 & 0.80 & 0.80 & 0.80 & 0.80 \\
\hline TY & 0.20 & 0.20 & 0.23 & 0.32 & 0.60 & 0.69 & 0.80 & 0.80 & 0.80 & 0.80 & 0.80 & 0.80 & 0.80 & 0.80 \\
\hline
\end{tabular}


Table 2. List of markets and corresponding sets of optimal weights for the AvDD problem. The solution achieving maximal reward/risk ratio is boldfaced.

\begin{tabular}{|l|l|l|l|l|l|l|l|l|l|l|l|l||}
\hline \hline Risk, \% & 0.77 & 1.00 & $\mathbf{1 . 2 3}$ & 1.46 & 1.50 & 1.69 & 1.92 & 2.15 & 2.38 & 2.61 & 2.84 & 3.07 \\
\hline Reward, \% & 21.7 & 35.6 & $\mathbf{4 5 . 3}$ & 53.3 & 54.5 & 59.9 & 65.7 & 70.6 & 74.8 & 78.2 & 81.2 & 83.0 \\
\hline Reward/risk & 28.2 & 35.6 & $\mathbf{3 6 . 8}$ & 36.5 & 36.3 & 35.4 & 34.2 & 32.9 & 31.4 & 30.0 & 28.6 & 27.0 \\
\hline \hline
\end{tabular}

OPTIMAL PORTFOLIO CONFIGURATION

\begin{tabular}{|c|c|c|c|c|c|c|c|c|c|c|c|c|}
\hline AAO & 0.20 & 0.46 & 0.61 & 0.77 & 0.80 & 0.80 & 0.80 & 0.80 & 0.80 & 0.80 & 0.80 & 0.80 \\
\hline$\overline{A D}$ & 0.21 & 0.57 & 0.80 & 0.80 & 0.80 & 0.80 & 0.80 & 0.80 & 0.80 & 0.80 & 0.80 & 0.80 \\
\hline$\overline{\mathrm{AXB}}$ & 0.20 & 0.20 & 0.23 & 0.55 & 0.62 & 0.80 & 0.80 & 0.80 & 0.80 & 0.80 & 0.80 & 0.80 \\
\hline$\overline{B D}$ & 0.20 & 0.20 & 0.20 & 0.20 & 0.20 & 0.20 & 0.20 & 0.20 & 0.20 & 0.52 & 0.80 & 0.80 \\
\hline$\overline{\mathbf{B P}}$ & 0.20 & 0.20 & 0.20 & 0.20 & 0.20 & 0.20 & 0.20 & 0.20 & 0.43 & 0.80 & 0.80 & 0.80 \\
\hline CD & 0.20 & 0.37 & 0.54 & 0.80 & 0.80 & 0.80 & 0.80 & 0.80 & 0.80 & 0.80 & 0.80 & 0.80 \\
\hline $\mathbf{C P}$ & 0.24 & 0.60 & 0.80 & 0.80 & 0.80 & 0.80 & 0.80 & 0.80 & 0.80 & 0.80 & 0.80 & 0.80 \\
\hline DGB & 0.33 & 0.80 & 0.80 & 0.80 & 0.80 & 0.80 & 0.80 & 0.80 & 0.80 & 0.80 & 0.80 & 0.80 \\
\hline DX & 0.20 & 0.20 & 0.20 & 0.20 & 0.20 & 0.20 & 0.20 & 0.20 & 0.20 & 0.30 & 0.71 & 0.80 \\
\hline ED & 0.20 & 0.30 & 0.35 & 0.33 & 0.32 & 0.21 & 0.31 & 0.44 & 0.70 & 0.75 & 0.80 & 0.80 \\
\hline EU & 0.20 & 0.20 & 0.20 & 0.20 & 0.20 & 0.20 & 0.46 & 0.80 & 0.80 & 0.80 & 0.80 & 0.80 \\
\hline FV & 0.20 & 0.20 & 0.37 & 0.50 & 0.53 & 0.76 & 0.80 & 0.80 & 0.80 & 0.80 & 0.80 & 0.80 \\
\hline FXADJY & 0.20 & 0.20 & 0.20 & 0.31 & 0.33 & 0.42 & 0.57 & 0.73 & 0.80 & 0.80 & 0.80 & 0.80 \\
\hline FXBPJY & 0.20 & 0.20 & 0.32 & 0.49 & 0.50 & 0.69 & 0.80 & 0.80 & 0.80 & 0.80 & 0.80 & 0.80 \\
\hline FXEUBP & 0.20 & 0.20 & 0.29 & 0.53 & 0.58 & 0.77 & 0.80 & 0.80 & 0.80 & 0.80 & 0.80 & 0.80 \\
\hline FXEUJY & 0.20 & 0.59 & 0.80 & 0.80 & 0.80 & 0.80 & 0.80 & 0.80 & 0.80 & 0.80 & 0.80 & 0.80 \\
\hline FXEUSF & 0.29 & 0.62 & 0.80 & 0.80 & 0.80 & 0.80 & 0.80 & 0.80 & 0.80 & 0.80 & 0.80 & 0.80 \\
\hline FXNZUS & 0.20 & 0.20 & 0.20 & 0.20 & 0.20 & 0.27 & 0.80 & 0.80 & 0.80 & 0.80 & 0.80 & 0.80 \\
\hline FXUSSG & 0.20 & 0.20 & 0.20 & 0.40 & 0.48 & 0.71 & 0.80 & 0.80 & 0.80 & 0.80 & 0.80 & 0.80 \\
\hline FXUSSK & 0.20 & 0.74 & 0.80 & 0.80 & 0.80 & 0.80 & 0.80 & 0.80 & 0.80 & 0.80 & 0.80 & 0.80 \\
\hline GC & 0.20 & 0.20 & 0.20 & 0.20 & 0.20 & 0.20 & 0.20 & 0.20 & 0.20 & 0.20 & 0.20 & 0.79 \\
\hline JY & 0.20 & 0.38 & 0.62 & 0.80 & 0.80 & 0.80 & 0.80 & 0.80 & 0.80 & 0.80 & 0.80 & 0.80 \\
\hline LBT & 0.20 & 0.52 & 0.80 & 0.80 & 0.80 & 0.80 & 0.80 & 0.80 & 0.80 & 0.80 & 0.80 & 0.80 \\
\hline LFT & 0.20 & 0.20 & 0.20 & 0.20 & 0.20 & 0.20 & 0.20 & 0.20 & 0.20 & 0.36 & 0.46 & 0.80 \\
\hline LGL & 0.20 & 0.20 & 0.20 & 0.20 & 0.20 & 0.20 & 0.29 & 0.48 & 0.65 & 0.80 & 0.80 & 0.80 \\
\hline LML & 0.20 & 0.20 & 0.21 & 0.34 & 0.34 & 0.49 & 0.64 & 0.80 & 0.80 & 0.80 & 0.80 & 0.80 \\
\hline MNN & 0.20 & 0.20 & 0.20 & 0.20 & 0.20 & 0.20 & 0.42 & 0.80 & 0.80 & 0.80 & 0.80 & 0.80 \\
\hline SF & 0.20 & 0.20 & 0.38 & 0.50 & 0.54 & 0.67 & 0.80 & 0.80 & 0.80 & 0.80 & 0.80 & 0.80 \\
\hline SI & 0.20 & 0.20 & 0.20 & 0.20 & 0.20 & 0.20 & 0.20 & 0.20 & 0.20 & 0.20 & 0.20 & 0.80 \\
\hline SJB & 0.23 & 0.67 & 0.80 & 0.80 & 0.80 & 0.80 & 0.80 & 0.80 & 0.80 & 0.80 & 0.80 & 0.80 \\
\hline SNI & 0.20 & 0.33 & 0.47 & 0.62 & 0.66 & 0.72 & 0.80 & 0.80 & 0.80 & 0.80 & 0.80 & 0.80 \\
\hline TY & 0.20 & 0.20 & 0.20 & 0.20 & 0.20 & 0.20 & 0.20 & 0.32 & 0.69 & 0.77 & 0.80 & 0.80 \\
\hline
\end{tabular}


Table 3. List of markets and corresponding sets of optimal weights for the CDaR problem with $(1-\alpha)=0.05$. The solution achieving maximal reward/risk ratio is boldfaced.

\begin{tabular}{|l|c|c|c|c|c|c|c|c|c|c|c|c|c|c||}
\hline Risk, \% & 3.0 & 3.2 & 3.7 & 3.8 & 3.9 & 4.0 & $\mathbf{5 . 0}$ & 6.0 & 7.0 & 8.0 & 9.0 & 10.0 & 11.0 & 12.0 \\
\hline Reward, \% & 24.2 & 27.2 & 33.3 & 34.4 & 35.5 & 36.6 & $\mathbf{4 6 . 3}$ & 54.7 & 62.1 & 68.4 & 73.9 & 78.6 & 82.0 & 83.0 \\
\hline$\zeta_{\alpha}, \%$ & 2.55 & 2.64 & 3.10 & 3.18 & 3.27 & 3.36 & $\mathbf{4 . 2 6}$ & 5.13 & 6.02 & 6.81 & 7.66 & 8.61 & 9.57 & 9.98 \\
\hline Reward/Risk & 8.06 & 8.50 & 8.99 & 9.04 & 9.09 & 9.14 & $\mathbf{9 . 2 6}$ & 9.12 & 8.86 & 8.55 & 8.21 & 7.86 & 7.45 & 6.92 \\
\hline
\end{tabular}

\section{OPTIMAL PORTFOLIO CONFIGURATION}

\begin{tabular}{|c|c|c|c|c|c|c|c|c|c|c|c|c|c|c|}
\hline AAO & 0.20 & 0.21 & 0.30 & 0.32 & 0.33 & 0.34 & 0.49 & 0.54 & 0.69 & 0.80 & 0.80 & 0.80 & 0.80 & 0.80 \\
\hline $\mathbf{A D}$ & 0.24 & 0.36 & 0.60 & 0.64 & 0.68 & 0.69 & 0.80 & 0.80 & 0.80 & 0.80 & 0.80 & 0.80 & 0.80 & 0.80 \\
\hline$\overline{\mathrm{AXB}}$ & 0.20 & 0.20 & 0.20 & 0.20 & 0.20 & 0.20 & 0.20 & 0.20 & 0.33 & 0.46 & 0.80 & 0.80 & 0.80 & 0.80 \\
\hline BD & 0.20 & 0.20 & 0.20 & 0.20 & 0.20 & 0.20 & 0.20 & 0.60 & 0.69 & 0.67 & 0.80 & 0.80 & 0.80 & 0.80 \\
\hline $\mathbf{B P}$ & 0.20 & 0.20 & 0.20 & 0.20 & 0.20 & 0.20 & 0.20 & 0.20 & 0.20 & 0.20 & 0.20 & 0.20 & 0.80 & 0.80 \\
\hline CD & 0.20 & 0.20 & 0.29 & 0.31 & 0.32 & 0.33 & 0.49 & 0.64 & 0.80 & 0.80 & 0.80 & 0.80 & 0.80 & 0.80 \\
\hline $\mathbf{C P}$ & 0.23 & 0.34 & 0.41 & 0.44 & 0.46 & 0.51 & 0.80 & 0.80 & 0.80 & 0.80 & 0.80 & 0.80 & 0.80 & 0.80 \\
\hline DGB & 0.50 & 0.71 & 0.80 & 0.80 & 0.80 & 0.80 & 0.80 & 0.80 & 0.80 & 0.80 & 0.80 & 0.80 & 0.80 & 0.80 \\
\hline DX & 0.20 & 0.20 & 0.20 & 0.20 & 0.20 & 0.20 & 0.20 & 0.20 & 0.20 & 0.20 & 0.31 & 0.80 & 0.80 & 0.80 \\
\hline ED & 0.20 & 0.20 & 0.20 & 0.20 & 0.20 & 0.20 & 0.26 & 0.27 & 0.31 & 0.28 & 0.28 & 0.48 & 0.64 & 0.80 \\
\hline $\mathbf{E U}$ & 0.20 & 0.20 & 0.23 & 0.26 & 0.30 & 0.31 & 0.80 & 0.80 & 0.80 & 0.80 & 0.80 & 0.80 & 0.80 & 0.80 \\
\hline FV & 0.20 & 0.20 & 0.20 & 0.23 & 0.25 & 0.30 & 0.47 & 0.47 & 0.56 & 0.73 & 0.80 & 0.80 & 0.80 & 0.80 \\
\hline FXADJY & 0.20 & 0.22 & 0.33 & 0.34 & 0.35 & 0.36 & 0.49 & 0.69 & 0.80 & 0.80 & 0.80 & 0.80 & 0.80 & 0.80 \\
\hline FXBPJY & 0.20 & 0.20 & 0.20 & 0.20 & 0.20 & 0.20 & 0.20 & 0.32 & 0.50 & 0.73 & 0.80 & 0.80 & 0.80 & 0.80 \\
\hline FXEUBP & 0.20 & 0.20 & 0.29 & 0.31 & 0.34 & 0.34 & 0.43 & 0.39 & 0.46 & 0.76 & 0.80 & 0.80 & 0.80 & 0.80 \\
\hline FXEUJY & 0.20 & 0.35 & 0.68 & 0.72 & 0.74 & 0.77 & 0.80 & 0.80 & 0.80 & 0.80 & 0.80 & 0.80 & 0.80 & 0.80 \\
\hline FXEUSF & 0.20 & 0.20 & 0.28 & 0.30 & 0.31 & 0.29 & 0.38 & 0.59 & 0.80 & 0.80 & 0.80 & 0.80 & 0.80 & 0.80 \\
\hline FXNZUS & 0.20 & 0.20 & 0.20 & 0.20 & 0.20 & 0.20 & 0.20 & 0.20 & 0.20 & 0.20 & 0.20 & 0.20 & 0.77 & 0.80 \\
\hline FXUSSG & 0.20 & 0.20 & 0.20 & 0.20 & 0.20 & 0.20 & 0.20 & 0.37 & 0.59 & 0.75 & 0.80 & 0.80 & 0.80 & 0.80 \\
\hline FXUSSK & 0.20 & 0.20 & 0.22 & 0.22 & 0.24 & 0.25 & 0.61 & 0.80 & 0.80 & 0.80 & 0.79 & 0.80 & 0.80 & 0.80 \\
\hline GC & 0.20 & 0.20 & 0.20 & 0.20 & 0.20 & 0.20 & 0.20 & 0.20 & 0.20 & 0.20 & 0.20 & 0.20 & 0.20 & 0.80 \\
\hline JY & 0.31 & 0.35 & 0.42 & 0.43 & 0.45 & 0.47 & 0.75 & 0.80 & 0.80 & 0.80 & 0.80 & 0.80 & 0.80 & 0.80 \\
\hline LBT & 0.20 & 0.20 & 0.20 & 0.20 & 0.20 & 0.20 & 0.47 & 0.80 & 0.80 & 0.80 & 0.80 & 0.80 & 0.80 & 0.80 \\
\hline LFT & 0.20 & 0.20 & 0.20 & 0.20 & 0.20 & 0.20 & 0.25 & 0.28 & 0.43 & 0.58 & 0.66 & 0.76 & 0.80 & 0.80 \\
\hline LGL & 0.20 & 0.20 & 0.20 & 0.20 & 0.20 & 0.20 & 0.20 & 0.20 & 0.20 & 0.20 & 0.27 & 0.66 & 0.80 & 0.80 \\
\hline LML & 0.20 & 0.20 & 0.20 & 0.20 & 0.20 & 0.20 & 0.20 & 0.20 & 0.31 & 0.52 & 0.69 & 0.74 & 0.80 & 0.80 \\
\hline MNN & 0.20 & 0.20 & 0.20 & 0.20 & 0.20 & 0.20 & 0.20 & 0.34 & 0.74 & 0.80 & 0.80 & 0.80 & 0.80 & 0.80 \\
\hline SF & 0.20 & 0.20 & 0.20 & 0.20 & 0.20 & 0.20 & 0.20 & 0.54 & 0.80 & 0.80 & 0.80 & 0.80 & 0.80 & 0.80 \\
\hline SI & 0.20 & 0.20 & 0.20 & 0.20 & 0.20 & 0.20 & 0.20 & 0.20 & 0.20 & 0.20 & 0.20 & 0.20 & 0.58 & 0.80 \\
\hline SJB & 0.47 & 0.57 & 0.71 & 0.74 & 0.77 & 0.80 & 0.80 & 0.80 & 0.80 & 0.80 & 0.80 & 0.80 & 0.80 & 0.80 \\
\hline SNI & 0.21 & 0.22 & 0.29 & 0.29 & 0.30 & 0.33 & 0.58 & 0.80 & 0.80 & 0.80 & 0.80 & 0.80 & 0.80 & 0.80 \\
\hline TY & 0.20 & 0.20 & 0.20 & 0.20 & 0.20 & 0.20 & 0.20 & 0.20 & 0.20 & 0.39 & 0.70 & 0.80 & 0.80 & 0.80 \\
\hline
\end{tabular}


Figure 1. Efficient frontier for the MaxDD problem (rate of return versus MaxDD).

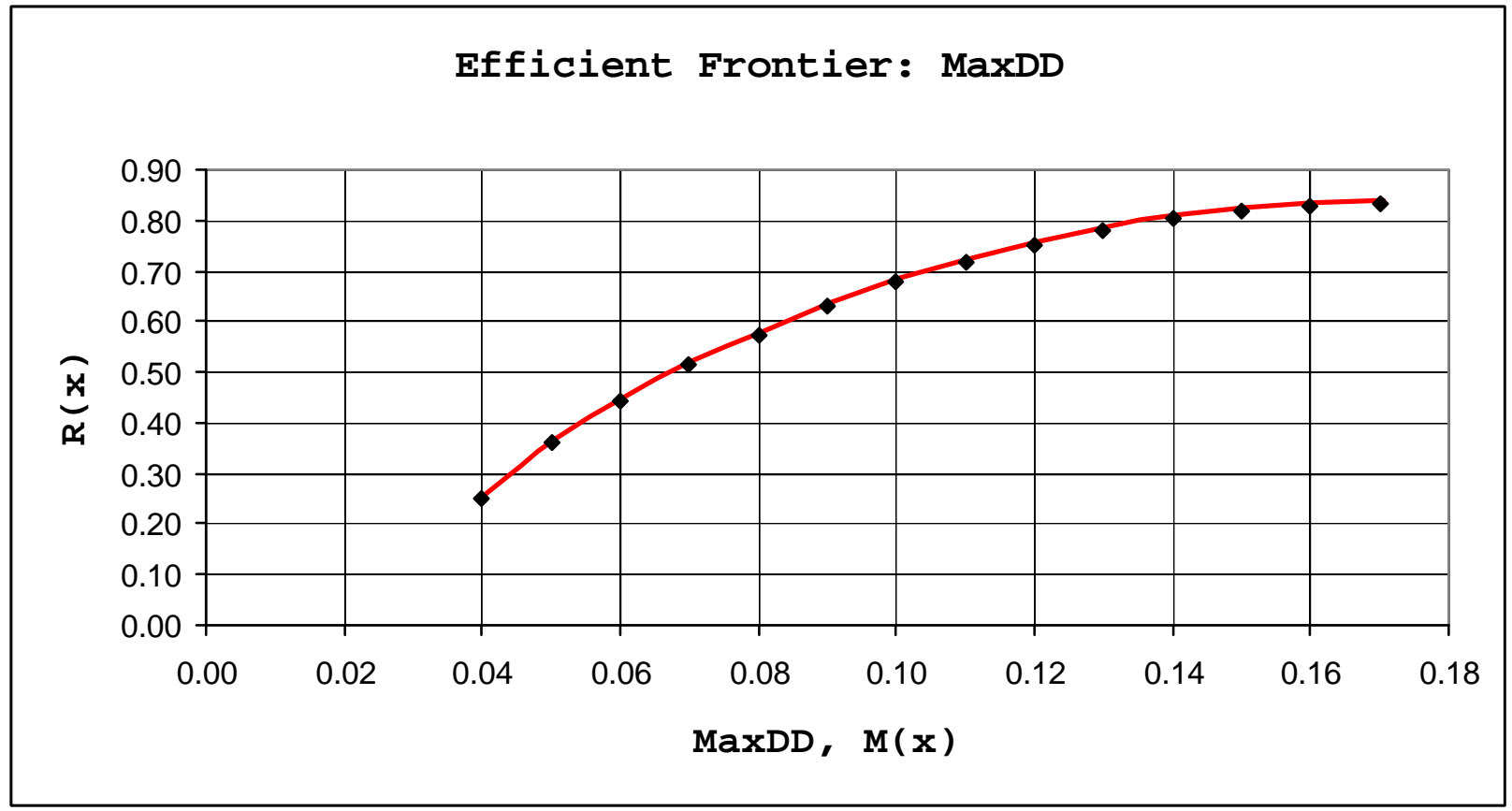

Figure 2. Efficient frontier for the AvDD problem (rate of return versus AvDD).

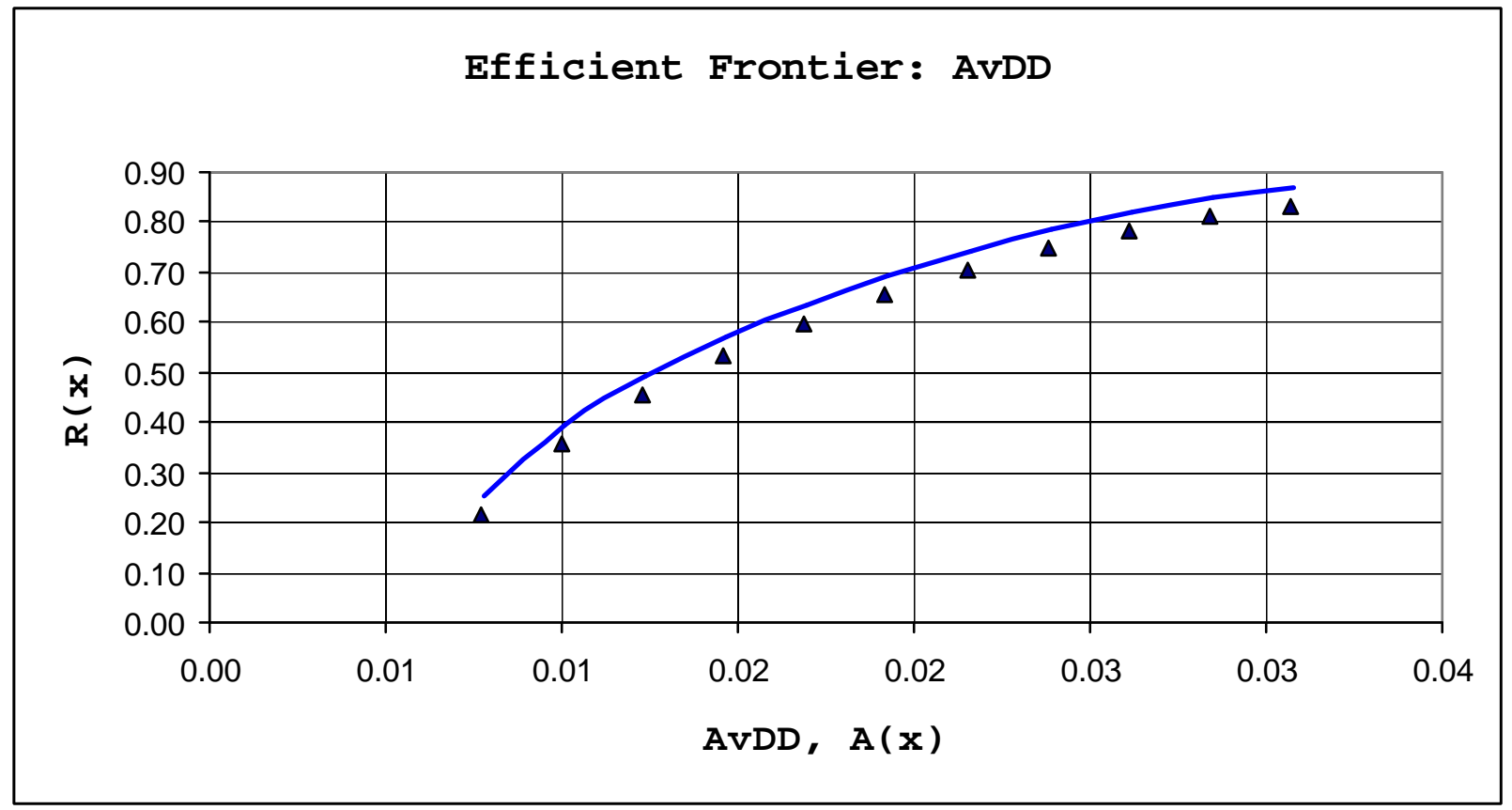


Figure 3. Reward-MaxDD graphs for optimal portfolios with $(1-\alpha)=0,0.05,0.4$ and $1 \mathrm{CDaR}$ constraints (rate of return versus MaxDD). The frontier is efficient only for the case with $(1-\alpha)=0 \mathrm{CDaR}$ constraints, which corresponds to the MaxDD risk measure.

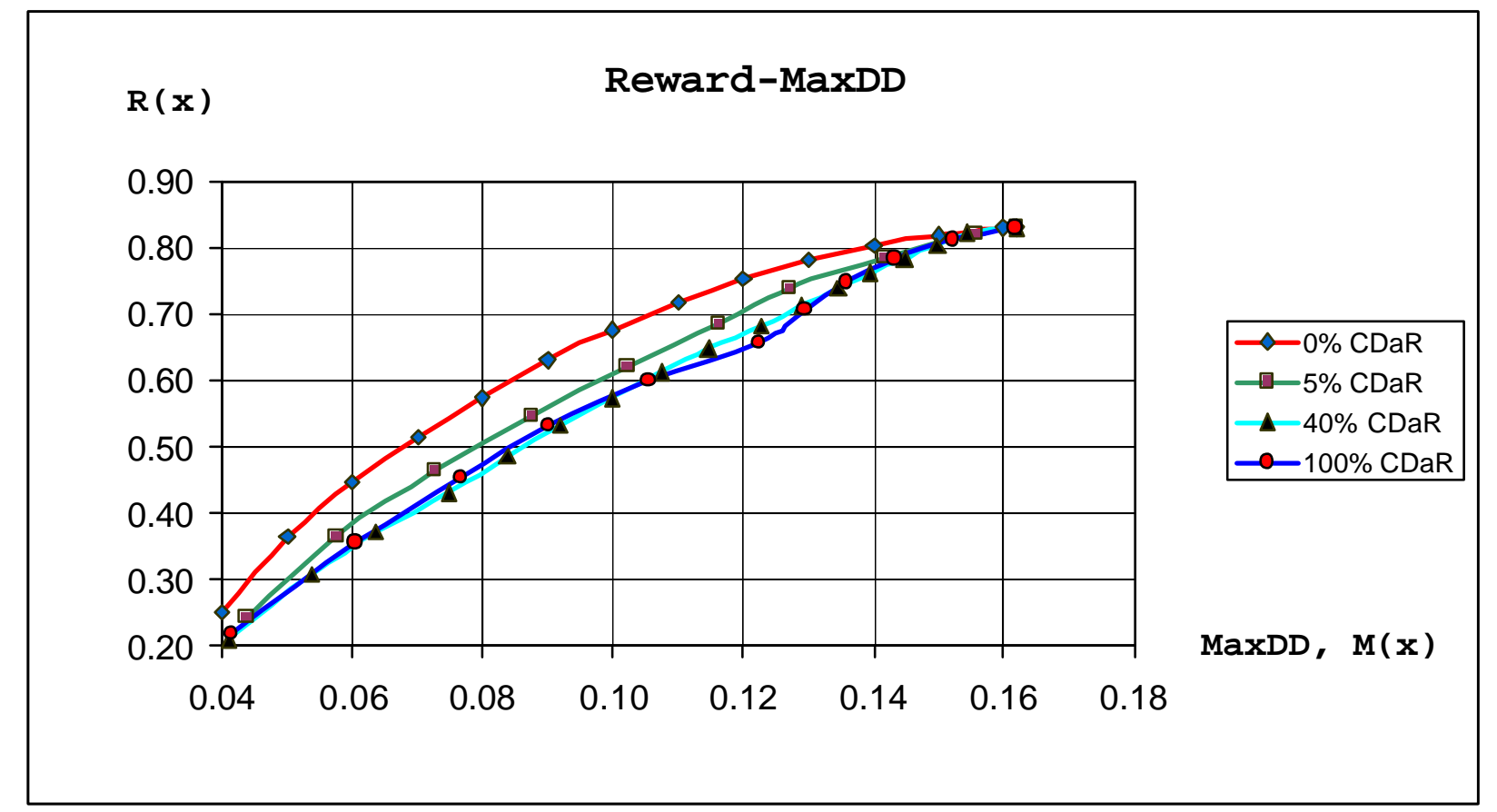

Figure 4. Reward-AvDD graphs for optimal portfolios with $(1-\alpha)=0,0.05,0.40$ and $1 \mathrm{CDaR}$ constraints (rate of return versus AvDD). The frontier is efficient only for the case with $(1-\alpha)=1 \mathrm{CDaR}$ constraints, which corresponds to the AvDD risk measure.

\section{$\mathbf{R}(\mathbf{x})$ \\ Reward-AvDD}

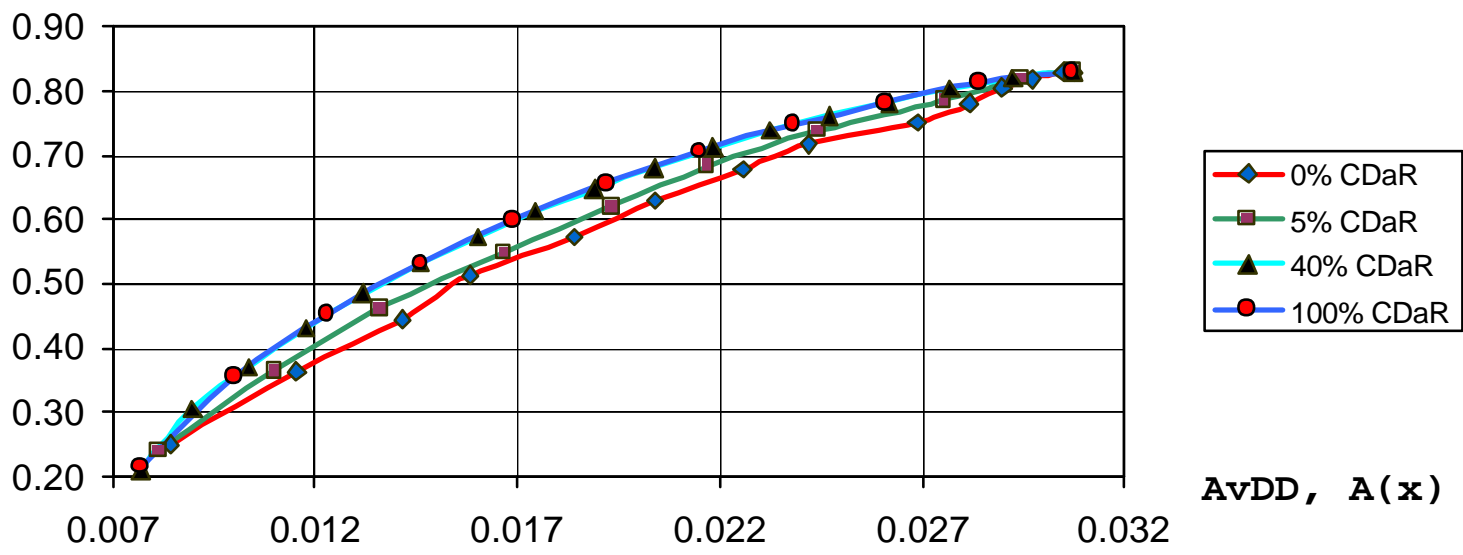


Figure 5. MaxDDRatio graphs for optimal portfolios with $(1-\alpha)=0,0.05,0.4$ and $1 \mathrm{CDaR}$ constraints (MaxDDRatio versus MaxDD). The maximum MaxDDRatio is achieved in the case with $(1-\alpha)=0 \mathrm{CDaR}$ constraints, which corresponds to the MaxDD risk measure.

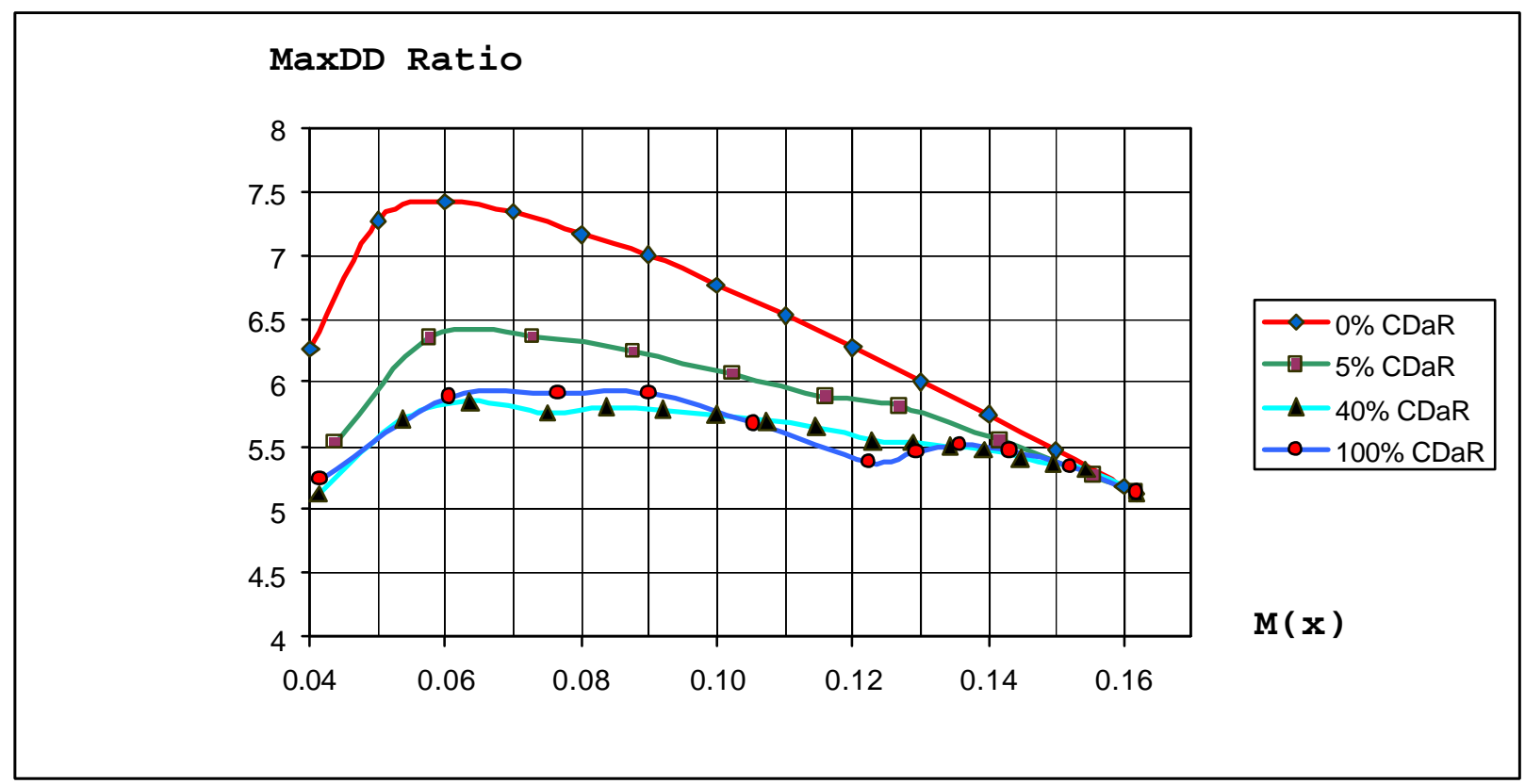

Figure 6. AvDDRatio graphs for optimal portfolios with $(1-\alpha)=0,0.05,0.40$ and $1 \mathrm{CDaR}$ constraints (AvDDRatio versus AvDD). The maximum AvDDRatio is achieved in the case with $(1-\alpha)=1 \mathrm{CDaR}$ constraints, which corresponds to the AvDD risk measure.

\section{AvDD Ratio}

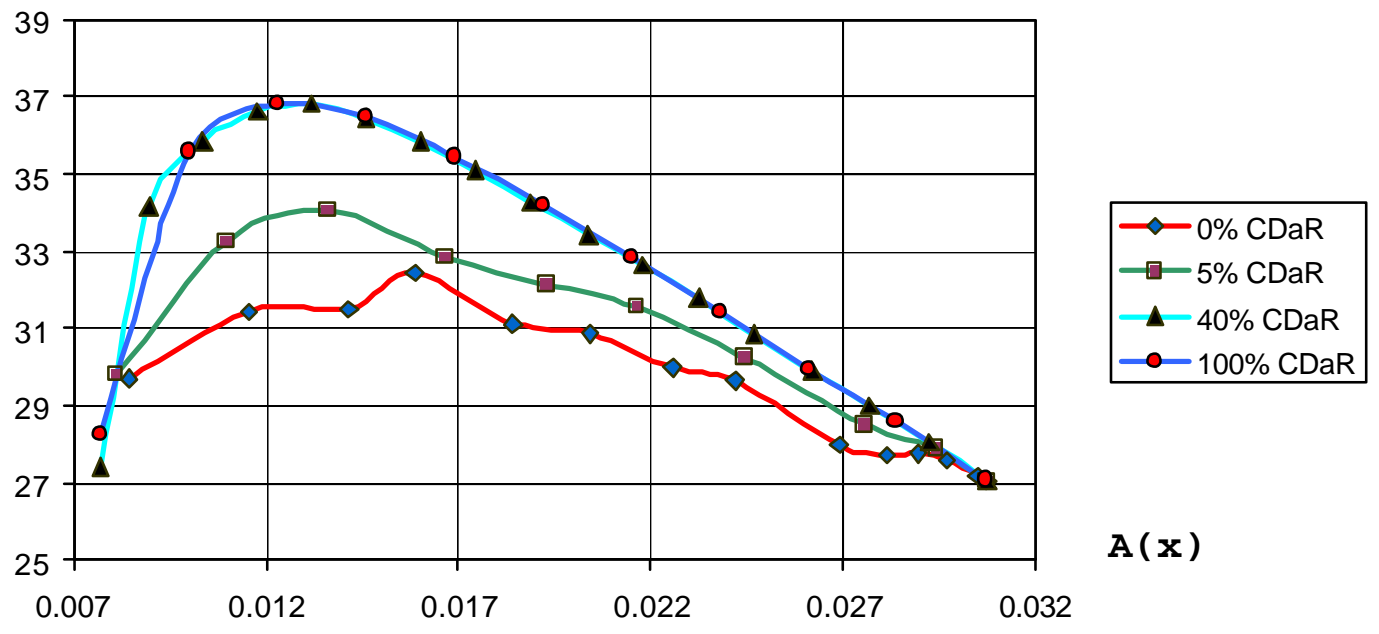




\section{Bibliography}

Andersson, F., Mausser, H., Rosen, D., and S. Uryasev, 2001, "Credit Risk Optimization with Conditional Value-At-Risk Criterion," Mathematical Programming, Series B 89, pp. 273-291.

Artzner, P., F. Delbaen, J. M. Eber and D. Heath, 1999, "Coherent Measures of Risk", Mathematical Finance 9, pp. 203-228.

Cvitanic, J., and I. Karatzas, 1995, "On Portfolio Optimization Under "Drawdown" Constraints", IMA Lecture Notes in Mathematics \& Applications 65, pp. 77-88.

Dembo, R. S., and A. J. King, 1992, "Tracking Models and the Optimal Regret Distribution in Asset Allocation", Applied Stochastic Models and Data Analysis 8, pp. 151-157.

Jorion, P., 1996, Value at Risk: A New Benchmark for Measuring Derivatives Risk (Irwin Professional Publisher).

Grinold, R. C., and R. N. Kahn, 1999, Active Portfolio Management (New York: McGrawHill).

Grossman, S. J., and Z. Zhou, 1993, "Optimal Investment Strategies for Controlling Drawdowns”, Mathematical Finance 3(3), pp. 241-276.

Konno, H., and H. Yamazaki, 1991, "Mean Absolute Deviation Portfolio Optimization Model and Its Application to Tokyo Stock Market”, Management Science 37, pp. 519-531.

Krokhmal, P., Palmquist, J., and S. Uryasev, 2002, "Portfolio Optimization with Conditional Value-At-Risk Objective and Constraints," The Journal of Risk 4(2).

Markowitz, H. M., 1952, “Portfolio Selection”, Journal of Finance 7(1), pp. 77-91.

Mausser, H., and D. Rosen, 1999, "Beyond VaR: From Measuring Risk to Managing Risk", ALGO Research Quarterly 1(2), pp. 5-20.

Rockafellar, R. T., 1970, Convex Analysis (Princeton University Press).

Rockafellar, R. T., and S. Uryasev, 2000, "Optimization of Conditional Value-at-Risk", The Journal of Risk 2, pp. 21-42.

Rockafellar, R. T., and S. Uryasev, 2002, "Conditional Value-at-Risk for General Loss Distributions", Journal of Banking and Finance 26, pp. 1443-1471.

Young, M. R., 1998, “A Minimax Portfolio Selection Rule with Linear Programming Solution”, Management Science 44(5), pp. 673-683.

Ziemba, W. T., and J. M. Mulvey, 1998, Worldwide Asset and Liability Modeling (Cambridge University Press). 